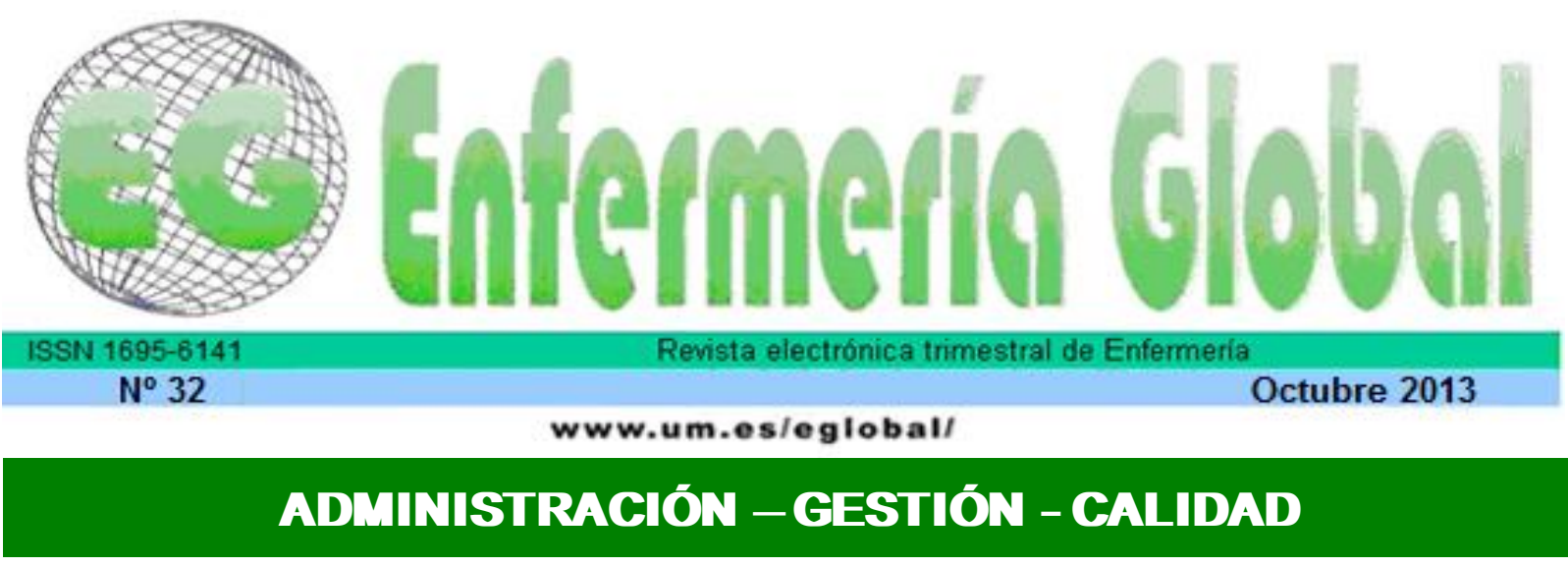

\title{
Pacientes por enfermera-auxiliar y presencia de eventos adversos en unidades de cuidados intensivos de Ibague-Colombia
}

Nurse-patient ratios or nurse assistant patient ratios and its relationship with the presence of adverse events in intensive care units of Ibague-Colombia

\section{*Lombo Caicedo, Jadith Cristina **Manrique Abril, Fred Gustavo}

*Magister en Enfermería. Profesora Asistente Cátedra. Universidad del Tolima. Ibague. E-mail: jclomboc@unal.edu.co ${ }^{* *}$ Enfermero, PhD Salud Pública. PhD(c) Investigación Clínica. Profesor Titular Universidad Nacional de Colombia- Grupo de Gerencia en Salud y Enfermería. Profesor Asociado Universidad Pedagógica y Tecnológica de Colombia.

Palabras clave: Odds-ratio; Relaciones enfermero-paciente; Eventos adversos; Atención dirigida al paciente; Seguridad del paciente.

Keywords: Odds ratio; Nurse-Patient Relations; Patient-Centered Care; Patient Safety.

\section{RESUMEN}

El personal necesario para atender pacientes influye en la calidad del servicio y es un indicador de resultados positivos o de eventos adversos durante la atención de pacientes en ambientes hospitalarios.

Objetivo: Establecer la relación existente entre el número de pacientes por enfermero(a)s y auxiliares de enfermería y la probabilidad de ocurrencia de eventos adversos durante un turno en tres unidades de cuidado intensivo de la ciudad de Ibague, Colombia.

Método: Estudio ecológico, longitudinal, en tres unidades de cuidado intensivo de lbague-Colombia en 1656 turnos observados.

Resultados: Hubo asociación entre la distribución de enfermero(a)s / paciente E/P y la probabilidad de incidencia de mortalidad 1/5. (OR:1.1) IC 95\%, paro cardiorrespiratorio.1/5 (OR 1.38) IC 95\% Infecciones derivadas de la atención en salud 1/5 (OR: 1.10) IC 95\%. Las dos primeras variables se estudiaron como generales debido a la limitación que impuso el subregistro evidenciado durante el tiempo del estudio. Se evidenció como única variable, de las analizadas en el estudio, sensiblemente relacionada con la razón de auxiliares de enfermería / paciente $A E / P$ la incidencia de escaras por decúbito 1/4 (OR:1.272) IC:95\%

Conclusiones: La razón enfermero(a) / paciente y auxiliar de enfermería / paciente se relacionan con eventos adversos sin significancia estadística, se hace necesario aumentar el número de observaciones y estudios para tener conclusiones sobre el número de pacientes por enfermera disminuyendo el riesgo de EA. 


\section{ABSTRACT}

The staff required to serve patients influences the quality of the service and is an indicator of positive or adverse events during patient care in hospital settings.

Objective: To establish the relationship between the Nurse-patient ratios and nurse assistants patient ratios and the probability of occurrence of adverse events during a shift of three intensive care units in Ibague, Colombia.

Methods: An ecological study, longitudinal, three intensive care units in 1656 lbague-Colombia shifts observed.

Results: There was an association between the Nurse-patient ratios and the probability of mortality incidence 1/5. (OR: 1.1) 95\%, unemployment cardiorrespiratorio.1 / 5 (OR 1.38) 95\% infections resulting from health care fifth (OR: 1.10) 95\%. The first two variables were studied as general because the limitation imposed by the underreporting demonstrated during the study period. It was shown that only variable, of those analyzed in the study, significantly related to the ratio of nurse assistants-patient ratio the incidence of pressure sores fourth (OR: 1.272) Cl: $95 \%$

Conclusions: The Nurse-patient ratio and nurse assistants-patient ratio adverse events are associated with no statistical significance, it is necessary to increase the number of observations and studies to draw strong conclusions about the number of patients per nurse decreasing the risk of $A D$.

\section{INTRODUCCIÓN}

Los eventos adversos (EA) se definen como acontecimientos relacionados con la atención recibida más que con la enfermedad subyacente que ocasionan fallecimiento, lesión, incapacidad o prolongación de la estancia hospitalaria de los pacientes. Los EA constituyen un problema de notable magnitud y trascendencia clínica, económica y legal, su estudio puede realizarse bien con carácter médico legal o bien con el fin de desarrollar políticas dirigidas a la mejora de la calidad asistencial y desarrollo de estrategias preventivas ${ }^{(1,2)}$.

Las Unidades de Cuidado Intensivo (UCls) son una de las áreas hospitalarias donde más eventos adversos se reportan, debido a las características de los pacientes, factores intrínsecos de los pacientes y las patologías por las cuales ingresan a las UCls, y extrínsecos como los múltiples procedimientos a los que son sometidos a diario, y a los múltiples dispositivos médicos invasivos para sostener la vida ${ }^{(3)}$.

En Estados Unidos, a finales de los 90, se inicia el desarrollo de estudios los cuales buscaban confirmar cómo la razón enfermero(a) paciente influía en los resultados de los pacientes en los hospitales; Aiken ${ }^{(4-9)}$ y Needleman ${ }^{(10,11)}$ en 799 hospitales de los Estados Unidos, usando modelos de regresión distintos concluyeron que hay una relación consistente y estrecha entre las variables relacionadas con el equipo de enfermería disponible y cinco resultados en los pacientes: Infecciones del tracto urinario, neumonía, tiempo de estancia, sangrado gastrointestinal alto y shock, en pacientes hospitalizados. En pacientes con cirugía mayor se encontró una relación estrecha y consistente entre la falla en el rescate y el equipo de enfermería y una relación más débil para neumonía e infección de vías urinarias.

Aiken ${ }^{(5)}$, en un estudio con 168 hospitales de Pennsylvania con 10.184 enfermeras y 232.342 pacientes de cirugía ortopédica, cirugía general, y cirugía vascular encontró dentro de los 30 días posteriores a la hospitalización que por cada paciente adicional por enfermera se asoció con un $7 \%$ (Odss Ratio $[\mathrm{OR}]=1,07$ ) la probabilidad de morir 
y aumento en las probabilidades de falla para rescate, en este estudio también encontró que por cada paciente adicional por enfermero(a) se asoció con un de $23 \%$ de aumento en la probabilidad de agotamiento y un $15 \%$ de aumento en la probabilidad de insatisfacción laboral.

Beglinger ${ }^{(12)}$, evidenció una disminución de estancia hospitalaria global en un $20.2 \%$ entre 1990 y 2005 al aumentar en un 27\% la intensidad en horas de atención de enfermería, así se disminuía un día de hospitalización, comprobando que a mayor cantidad de horas de enfermería, menos días de hospitalización. Se encontró también que las condiciones y capacidades del personal impactó sobre la calidad del servicio prestado a los pacientes.

El estudio SYREC ${ }^{(3)}$ encontró que en los eventos adversos en cuidado Intensivo existían una serie de Factores Contribuyentes (FC) donde de los 1424 incidentes notificados, 1247 presentaban algún $\mathrm{FC}$, y se refieren principalmente a la sobrecarga asistencial. El factor que más veces se ha notificado en este grupo relacionado con incidentes ha sido la excesiva carga de trabajo, 34.63\%, y a su alrededor, la presión del tiempo, 19.43\%, la razón de personal deficiente, $8.95 \%$, y la fatiga ligada a turnos, $7.60 \%$. En un entorno similar, el personal temporal, $11.15 \%$, y la falta de habilidades, $6.25 \%$.

Actualmente en relación con estos factores contribuyentes se encuentran estudios que apuntan hacia investigaciones relacionadas, con la REP y su relación con la mortalidad en cuidado intensivo y las complicaciones secundarias a la hospitalización ${ }^{(4,6,13,14)}$.

La razón enfermero(a) / paciente (REP) es el número de pacientes asignados a cada enfermero(a) para brindar cuidados durante un periodo de tiempo (15-17). La razón auxiliar de enfermería / paciente (RAEP) corresponde a la relación que se establece entre el número de pacientes que le son asignados a un auxiliar de enfermería entendiéndose por auxiliar de enfermería la persona que recibe capacitación en instituciones educativas reguladas por el gobierno pero no universitarias.

El objetivo de esta investigación fue establecer la posible asociación de la REP y RAEP y la probabilidad de ocurrencia de eventos adversos en tres unidades de cuidado intensivo en la ciudad de Ibagué, Colombia.

\section{MÉTODO}

Este estudio es de carácter epidemiológico y corresponde a un estudio ecológico(18, 19), se tomó como unidad de observación el turno (jornada laboral de 6 o 12 horas), una misma área en diferentes periodos de tiempo que para este caso se denominara, tres unidades de cuidados intensivos observadas durante 6 meses, 1656 turnos observados.

Se construyó un formulario para recolectar los datos en SPSS $11.5 \AA$ con las variables número de enfermeras, numero de auxiliares, pacientes, egresos, ingresos, y las variables de salida infecciones derivadas de la atención en salud, caídas, escaras o úlceras por presión por decúbito, error en el suministro de medicamentos, desplazamiento de dispositivos médicos, paro y muerte. 
Se realiza análisis descriptivo y se construyen tablas de doble entrada estableciendo como medida de asociación los Odss Ratio (OR) con intervalos de confianza en 95\% y prueba exacta de Fisher; se exploró la influencia de co-variables durante los turnos observados en las unidades de cuidado intensivo que pueden comportarse como intervinientes o confusoras en la razón enfermero(a) /paciente y auxiliar de enfermería / paciente y la probabilidad de ocurrencia de los eventos adversos.

El estudio fue aprobado por el Comité de Ética de la Facultad de Enfermería de la Universidad Nacional de Colombia y por cada institución participante.

\section{RESULTADOS}

La asignación de pacientes por personal de enfermería fue: para el caso de los profesionales, en casi el $99 \%$ de los turnos les fueron asignados entre cuatro y seis pacientes, mientras que a las auxiliares les asignaron en el 93,4\% de los turnos 3 o 4 pacientes.

Tabla 1. Distribución paciente por personal de enfermería en 1656 turnos en tres unidades de cuidado intensivo de lbague

\begin{tabular}{|l|l|l|}
\hline REP & FRECUENCIA & PORCENTAJE \\
\hline $1 \mathrm{~A} 2$ & 15 & $0,9 \%$ \\
\hline$>2 \leq 4$ & 808 & $48,8 \%$ \\
\hline$>4 \leq 6$ & 827 & $49,93 \%$ \\
\hline$>6$ & 6 & $0,36 \%$ \\
\hline RAEP & FRECUENCIA & PORCENTAJE \\
\hline $1-2$ & 74 & $4,4 \%$ \\
\hline$>2 \leq 4$ & 1547 & $93,4 \%$ \\
\hline$>4-6$ & 90 & $5,43 \%$ \\
\hline
\end{tabular}

De los 1656 turnos observados, se presentó algún evento adverso en el $31.5 \%$ de los turnos. El principal evento observado fue la muerte seguido de los paros cardiorrespiratorios. Tabla 2.

Tabla 2. Distribución porcentual de los eventos adversos presentados en las Unidades de Cuidado Intensivos

\begin{tabular}{|l|l|l|l|l|l|l|}
\hline EA & INFECCIONES & $\begin{array}{l}\text { DESPLAZA } \\
\text { MIENTO } \\
\text { DE } \\
\text { DISPOSITI } \\
\text { VOS }\end{array}$ & $\begin{array}{l}\text { ESCAR } \\
\text { AS }\end{array}$ & $\begin{array}{l}\text { ERROR EN LA } \\
\text { ADMINISTRA- } \\
\text { CIÓN DE } \\
\text { MEDICAMEN- } \\
\text { TOS }\end{array}$ & $\begin{array}{l}\text { MUER } \\
\text { TES }\end{array}$ & PAROS \\
\hline NÚMEROS & 61 & 77 & 43 & 4 & 188 & 149 \\
\hline $\begin{array}{l}\text { PORCENTA- } \\
\text { JES }\end{array}$ & $11,7 \%$ & $14,75 \%$ & $8,24 \%$ & $0,77 \%$ & $36,0 \%$ & $28,5 \%$ \\
\hline
\end{tabular}


Las infecciones y el desplazamiento de dispositivos médicos fue escaso, pero importante.

Tabla 3. Distribución porcentual de infecciones relacionadas con la atención en salud

\begin{tabular}{|c|c|c|c|c|c|c|c|}
\hline EA & \multicolumn{2}{|l|}{ Flebitis } & Bacteriemias & \multicolumn{2}{|c|}{ Neumonías } & \multicolumn{2}{|l|}{ IVU } \\
\hline Números & \multicolumn{2}{|l|}{9} & 5 & \multicolumn{2}{|l|}{26} & \multicolumn{2}{|l|}{21} \\
\hline \multirow[t]{2}{*}{ Porcentajes } & \multicolumn{2}{|l|}{$14,75 \%$} & $8,20 \%$ & \multicolumn{2}{|l|}{$42,62 \%$} & \multicolumn{2}{|l|}{$34,43 \%$} \\
\hline & $\begin{array}{l}\text { Acceso } \\
\text { venoso } \\
\text { periferico }\end{array}$ & $\begin{array}{l}\text { Catéter } \\
\text { central }\end{array}$ & $\begin{array}{l}\text { Desplazamiento } \\
\text { de } \\
\text { ventriculostomía }\end{array}$ & SOG & Sv & TOT & $\begin{array}{l}\text { Tubo } \\
\text { a } \\
\text { torax }\end{array}$ \\
\hline NUMEROS & 9 & 8 & 1 & 29 & 7 & 20 & 3 \\
\hline PORCENTAJES & $11,69 \%$ & $10,39 \%$ & $1,30 \%$ & $37,66 \%$ & $9,09 \%$ & $25,97 \%$ & $3,90 \%$ \\
\hline
\end{tabular}

SOG: Sonda orogástrica; SV: Sonda vesical; TOT: Tubo oro traqueal; IVU: infección de vías urinarias. (fuente: los autores)

La neumonía la infección con mayor frecuencia dentro del estudio $42.62 \%$, seguida de infección de vías urinarias $34.43 \%$ e infecciones en catéter periférico $14.75 \%$, en menor porcentaje $8.20 \%$ se presentan bacteriemias asociadas a catéter central.

Usando el OR se exploraron diferentes puntos de corte acorde con el número de pacientes asignados por enfermera en cada turno y se definió el resultado en términos presencia de mortalidad durante el turno, paro, infección, escaras.

En las tablas 4, 5 y 6 se observa cómo el factor protector para los eventos adversos se encuentra asignando menos de 4 pacientes por enfermera y se vuelve riesgo al asignar un paciente adicional, para las variables de resultado estudiadas.

Tabla 4.REP y su relación con la probabilidad de incidencia de muerte.

\begin{tabular}{|c|c|c|c|c|c|}
\hline REP & & SI & $\mathrm{NO}$ & OR (IC95\%) & $\begin{array}{ll}P & (\mathrm{CHI} \\
2) & \\
\end{array}$ \\
\hline \multirow{2}{*}{$2 \mathrm{P} / \mathrm{E}$} & $\mathrm{SI}$ & 1 & 14 & \multirow{2}{*}{$\begin{array}{l}0,5554 \\
(0,07264,2478)\end{array}$} & 0,56 \\
\hline & $\mathrm{NO}$ & 187 & 1454 & & \\
\hline \multirow{2}{*}{$3 P / E$} & SI & 13 & 127 & \multirow{2}{*}{$\begin{array}{l}0,7844 \\
(0,43391,4180)\end{array}$} & 0,42 \\
\hline & NO & 175 & 1341 & & \\
\hline \multirow{2}{*}{$4 \mathrm{P} / \mathrm{E}$} & SI & 90 & 733 & \multirow{2}{*}{$\begin{array}{l}0,9209 \\
(0,67951,2479)\end{array}$} & 0,59 \\
\hline & NO & 98 & 735 & & \\
\hline \multirow{2}{*}{$5 \mathrm{P} / \mathrm{E}$} & $\mathrm{SI}$ & 155 & 1187 & \multirow{2}{*}{$\begin{array}{l}1,1119 \\
(0,7471 \quad 1,6549)\end{array}$} & 0,6 \\
\hline & $\mathrm{NO}$ & 33 & 281 & & \\
\hline
\end{tabular}

P/E: pacientes por enfermera; OR: Odds ratio; P:chi cuadrado -test exacto de Fisher. (Fuente: los autores) 
Tabla 5. REP y su relación con la probabilidad de paro en tres unidades de cuidado intensivo de la ciudad de lbagué.

\begin{tabular}{|c|c|c|c|c|c|}
\hline REP & & $\mathrm{SI}$ & $\mathrm{NO}$ & OR (IC95\%) & $\mathrm{P}(\mathrm{CHI} 2)$ \\
\hline \multirow[t]{2}{*}{$2 \mathrm{P} / \mathrm{E}$} & $\mathrm{SI}$ & 1 & 14 & \multirow{2}{*}{$\begin{array}{l}0.72 \\
(0,09-5,5)\end{array}$} & \multirow[t]{2}{*}{0,7513} \\
\hline & $\mathrm{NO}$ & 148 & 1493 & & \\
\hline \multirow[t]{2}{*}{$3 \mathrm{P} / \mathrm{E}$} & $\mathrm{SI}$ & 10 & 130 & \multirow{2}{*}{$\begin{array}{l}0.76 \\
(0,39-1,5)\end{array}$} & \multirow[t]{2}{*}{0,4229} \\
\hline & $\mathrm{NO}$ & 139 & 1377 & & \\
\hline \multirow[t]{2}{*}{$4 \mathrm{P} / \mathrm{E}$} & $\mathrm{SI}$ & 73 & 750 & \multirow{2}{*}{$\begin{array}{l}0.96 \\
(0,69-1,3)\end{array}$} & \multirow[t]{2}{*}{0,8569} \\
\hline & $\mathrm{NO}$ & 76 & 757 & & \\
\hline \multirow[t]{2}{*}{$5 \mathrm{P} / \mathrm{E}$} & SI & 127 & 1215 & \multirow{2}{*}{$\begin{array}{l}1,38 \\
(0.86-2,22)\end{array}$} & \multirow[t]{2}{*}{0,1708} \\
\hline & $\mathrm{NO}$ & 22 & 292 & & \\
\hline
\end{tabular}

P/E: pacientes por enfermera; OR: odds ratio; P:chi cuadrado -test exacto de Fisher. (Fuente: los autores)

Se percibe cómo existe un aumento gradual del riesgo (tendencia y colinealidad) relacionado con el aumento en el número de pacientes por enfermera para la probabilidad de paro cardiorrespiratorio en la $\mathrm{UCl}$, aunque no es estadísticamente significativo es un hallazgo importante para la gestión de recurso humano y el desarrollo gerencial dentro de las UCls estudiadas.

Tabla 6. REP y su relación con las infecciones derivadas de la atención en salud.

\begin{tabular}{|c|c|c|c|c|c|}
\hline REP & & SI & NO & OR (IC95\%) & $\mathrm{P}(\mathrm{CHI} 2)$ \\
\hline \multirow[b]{2}{*}{$2 \mathrm{P} / \mathrm{E}$} & SI & 0 & 15 & \multirow{2}{*}{$\mathrm{NC}$} & \multirow{2}{*}{0,4627} \\
\hline & NO & 57 & 1584 & & \\
\hline \multirow{2}{*}{ 3P/E } & SI & 3 & 137 & \multirow{2}{*}{$\begin{array}{l}0,5929 \\
(0,1830-1,9212)\end{array}$} & \multirow{2}{*}{0,3783} \\
\hline & NO & 54 & 1462 & & \\
\hline \multirow{2}{*}{$4 \mathrm{P} / \mathrm{E}$} & SI & 27 & 796 & \multirow{2}{*}{$\begin{array}{l}0,9079 \\
(0,5349-1,5411)\end{array}$} & \multirow{2}{*}{0,7204} \\
\hline & $\mathrm{NO}$ & 30 & 803 & & \\
\hline \multirow{2}{*}{$5 \mathrm{P} / \mathrm{E}$} & SI & 47 & 1295 & \multirow{2}{*}{$\begin{array}{l}1,1033 \\
(0,5512-2,2083)\end{array}$} & \multirow{2}{*}{0,7812} \\
\hline & $\mathrm{NO}$ & 10 & 304 & & \\
\hline
\end{tabular}

P/E: pacientes por enfermera; OR: Odds ratio; P:chi cuadrado -test exacto de Fisher. (Fuente: los autores)

Se puede evidenciar el aumento del riesgo de incidencia de las infecciones derivadas de la atención en salud, cuando al enfermero(a) se le asignan 5 pacientes críticamente enfermos. Es notable el cambio de factor protector a riesgo tanto para la probabilidad de muerte, paro e infección cuando se asignan más de 4 pacientes a enfermera.

La RAEP y la probabilidad de incidencia de los EA se exploraron con cada una de las variables de resultado propuestas; muerte, paro, infecciones derivadas de la atención, desplazamiento de dispositivos médicos, de las variables de resultado estudiadas y solamente la presencia de escaras fue la variable en la cual se encontró una posible relación. 
Tabla 7. RAEP y su relación con la presencia de escaras en tres unidades de cuidado intensivo de la ciudad de lbague

\begin{tabular}{|l|l|l|l|l|l|}
\hline RAEP & & $\mathrm{SI}$ & $\mathrm{NO}$ & $\mathrm{OR}(\mathrm{IC95} \%)$ & $\mathrm{P}(\mathrm{CHI} 2)$ \\
\hline $2 \mathrm{P} / \mathrm{aE}$ & $\mathrm{SI}$ & 1 & 73 & 0,4679 & 0,4450 \\
\cline { 2 - 5 } & $\mathrm{NO}$ & 45 & 1537 & $(0,0636-3,4418)$ & \\
\hline \multirow{3}{*}{$3 \mathrm{P} / \mathrm{aE}$} & $\mathrm{SI}$ & 24 & 913 & 0,8328 & 0,548 \\
\cline { 2 - 5 } & $\mathrm{NO}$ & 22 & 697 & $(0,4631-1,4977)$ & \\
\hline \multirow{2}{*}{$4 \mathrm{P} / \mathrm{aE}$} & $\mathrm{SI}$ & 44 & 1522 & 1,272 & 0,7415 \\
& $\mathrm{NO}$ & 2 & 88 & $(0,3034-5,3328)$ & \\
\hline $5 \mathrm{P} / \mathrm{aE}$ & $\mathrm{SI}$ & 46 & 1602 & $\mathrm{NC}$ & 0,6318 \\
\cline { 2 - 5 } & $\mathrm{NO}$ & 0 & 8 & & \\
\hline
\end{tabular}

P/aE: pacientes por Auxiliar de enfermería; OR: Odds ratio; P:chi cuadrado -test exacto de Fisher. (Fuente: los autores)

Aquí al igual que en el personal de enfermeras profesionales, la presencia de escaras se relaciona con un factor de riesgo al asignar más de 3 pacientes por auxiliar de enfermería.

Al explorar las co-variables se pudo establecer que: la razón enfermero(a) / paciente y su relación con la probabilidad de muerte según los días de la semana se encontró que el día sábado se comporta como un día con un aumento en el riesgo de mortalidad cuando se establece una aumento en la razón de pacientes por enfermero(a) de 4 a 5 aumentando el OR de 0,7 a 1,1.

Para la predicción de muerte según los turnos; el turno de la mañana se muestra protector asignando menos de 4 pacientes por enfermera con $O R=0,49 ;(p<0,05)$, esta relación estadísticamente significativa.

Para otras variables que podrían comportarse como confusoras se estudiaron los egresos y los ingresos, encontrando que los egresos no modifican ni potencian los riesgos estudiados, mientras que los ingresos muestran una relación intervienen en el efecto frente a la probabilidad de presentar muertes en los turnos; en la cual una enfermero(a) con 5 pacientes y en presencia de ingresos aumenta el riesgo de mortalidad con un OR de 1.082.

La ausencia de egresos muestran una relación protectora para la incidencia de infecciones derivadas de la atención en salud hasta una razón paciente /enfermero(a) de 3 pacientes críticamente enfermos asignados a una enfermero(a) con un OR 0,93.

Se puede establecer una relación entre la incidencia de infecciones derivadas de la atención en salud y los ingresos en las tres unidades de cuidados intensivos, conservándose una relación protectora limítrofe hasta una paciente/enfermero de 5 con un OR de 0,96(IC de $95 \% \mathrm{chi}^{2}$ de 0.93). Los anteriores resultados se pueden dar debido a que cuanto más tiempo esté un paciente en una unidad de cuidados intensivos mayor es el riesgo de adquirir infecciones por el tiempo de exposición al riesgo de infecciones cruzadas y al monitoreo invasivo.

\section{DISCUSIÓN}

Para los análisis de recurso humano en salud y especialmente en enfermería en UCI, debe ser diferenciador, ya que la formación profesional versus la técnica predicen 
eventos adversos de forma disímil como en el presente estudio; es así como el estudio Syrec ${ }^{(3)}$ y uno realizado en Cuba ${ }^{(14)}$, demuestran cómo la existencia de un factor contribuyente potencia la incidencia de eventos adversos en el $87.7 \%$ de los casos reportados. Entre estos factores tienen importancia las cualidades de los diferentes profesionales con título universitario 0 no y que atienden pacientes críticamente enfermos. Eso también había sido establecido en Australia ${ }^{13)}$ donde notaron que no solamente se relacionaba una razón (un número) enfermero/paciente, sino que también las capacidades o cualidades académicas del personal de enfermería (el saber) se evidenciaron como fundamentales para garantizar la calidad de la atención, ya que no solo se estableció una relación con respecto al número de personas que dan atención.

Aiken ${ }^{(4)}$ encontró que la preparación educativa de los enfermeros era un importante factor que influía en la mortalidad del paciente: esto no se observó en este estudio, por cuanto no hubo significancia estadística en las asociaciones $(p>0,05)$. Sin embargo, esto sugiere la necesidad de plantear otras medidas de estructura como el "skill mix" o mezcla de habilidades para la asignación de personal, por cuanto esta medida apunta a denotar la importancia o no que tiene la diferencia en la formación

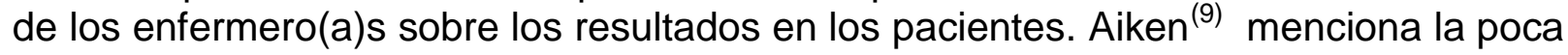
evidencia en la literatura respecto a la influencia del personal auxiliar de enfermería en los resultados negativos para los pacientes o que aumenten la carga de trabajo de los profesionales, hallazgo similar a lo encontrado en el presente estudio.

El estudio Syrec ${ }^{(3)}$ realizado en España con 79 unidades de cuidado intensivo encontró que la REP es de 2 pacientes por enfermero(a) para hospitales pequeños y grandes mientras que para hospitales medianos es de 1,83 pacientes por enfermero(a). Otro estudio realizado por Aiken ${ }^{(9)}$, estableció que la REP en California (USA) se encuentra en 2 pacientes para 1 enfermero(a) en las unidades de cuidado intensivo proporción que se cumple en el $85 \%$ de las unidades de cuidado intensivo de este estado. En Pennsylvania y New Jersey(5, 8, 10, 17) la REP de 1 enfermero(a) por dos pacientes críticos se cumple en el $61 \%$ y $73 \%$ respectivamente. En los resultados encontrados para este estudio se encontró una REP por arriba de 4 pacientes en el $71 \%$ de los turnos observados, alcanzándose una razón de hasta 11 pacientes por enfermero(a). Los turnos en los cuales la razón se asemeja al estándar propuesto para las Unidades de Cuidados Intensivos de California alcanzan a ser solamente el $2.7 \%$ de los observados, situación que baja en desventaja dentro de la calidad ofrecida en las UCI de Ibague-Colombia.

Una revisión de la literatura realizada en España ${ }^{(20)}$ para los auxiliares de enfermería muestra que la razón de camas por auxiliar en el $73 \%$ de las unidades de cuidado intensivo estudiadas fue de 3-4 pacientes, mientras que en esta investigación en cuanto a la RAEP se encuentra que el $93.4 \%$ de los turnos es mayor de dos y menor o igual a 4,siendo más alta que la referida en España.

El estudio Harvard Medical Practice II, (21) menciona que los errores humanos son los causantes de las dos terceras partes de las complicaciones en las unidades de cuidados intensivos. Estas complicaciones contribuyen a la mortalidad hospitalaria.

La REP es uno de los factores fundamentales que se ha relacionado con la morbimortalidad y la seguridad de los pacientes en las unidades de cuidado intensivo. Cuanto más elevada sea esta razón, mayor es el riesgo de sufrir un evento adverso y hasta la muerte ${ }^{(22-24)}$. Una revisión teórica realizada a 43 estudios ${ }^{(17)}$ sobre la REP y 
sus efectos sobre el paciente, llega a conclusiones símiles con algunos aspectos del presente estudio: una mayor REP tiene una relación inversa con la mortalidad, no se establece una relación entre el personal de enfermería y la incidencia de úlceras por presión y caídas de pacientes, al igual que en el presente trabajo, en el cual solo se establece una relación entre la RAEP y la presencia de escaras.

Un estudio realizado en Israel ${ }^{(25)}$, retoma los resultados de un estudio de Aiken con 168 hospitales donde mostró que un aumento en la REP de 1:4 a 1:6 lleva a un aumento en la mortalidad del 7\% y que aumentar la REP 1:4 a 1:8 aumenta la tasa de mortalidad de un $14 \%$. Para efectos del presente estudio aumentar la REP en una unidad de cuidados intensivos de 1:4 a 1:5 significa el aumento del OR de 0.92 a 1.1 lo cual implica que hasta una razón máxima de 4 pacientes por enfermero se establece como protectora para la variable muerte en pacientes críticamente enfermos.

Needleman (10) y Garretson (26) establecen una relación entre las infecciones derivadas de la atención en salud y la REP. La neumonía asociada a la ventilación mecánica es el evento adverso con mayor incidencia (42.62\%), valor similar al del estudio español con un $44.83 \%$, mientras que las infecciones en vías urinarias relacionadas a sonda uretral fueron $34.43 \%$ en comparación con el $18,97 \%$ del estudio SYREC (3), con una diferencia de $15,46 \%$.

Los de indicadores sensibles a la atención de enfermería permiten establecer que los indicadores más sensibles son paro cardiorrespiratorio, neumonía, infección del tracto urinario, úlceras por decúbito ${ }^{(10)}$. En esta investigación se encontró relación probable entre la REP y las variables de muerte, paro cardiorrespiratorio y/o shock, infecciones derivadas de la atención en salud. En las RAEP las variables sensibles son las escaras.

La REP y la incidencia de EA, se encuentran influenciadas por algunas variables como los ingresos los cuales influyen en el aumento de riesgo de muerte, mientras los no egresos influyen en el aumento de riesgo de infección. Aiken ${ }^{(6)}$, establece la influencia del entorno en la incidencia de los eventos adversos. Esto sugiere la necesidad de continuar desarrollando investigaciones sobre la manera como el contexto en el cual se da la atención de enfermería interviene en los procesos de calidad de la atención.

Existen otros factores que influyen en la REP-RAEP con probabilidad de ocurrencia de los eventos adversos como los ingresos para el riesgo de aumento de mortalidad y los no egresos para el aumento del riesgo de infecciones asociadas con la atención en salud.

Es necesario seguir estudiando lal carga laboral en la asignación de pacientes por enfermera y auxiliar de enfermería y los resultados en la atención en salud, así mismo explorar asociaciones con la presencia de los demás miembros del equipo de salud, los recursos físicos y económicos asignados, el estado del paciente, el nivel de resolución en tecnología y acceso y demás variables relacionadas con el sistema de salud y el modelo gerencial aplicado en cada institución entre otras tantas variables.

Aunque muchos de los resultados son apoyados con estudios internacionales, el número de pacientes asignados al profesional y auxiliar descritos en el presente estudio deben ser considerados con mucho cuidado en cada institución, ya que las 
limitaciones de la muestra y las condiciones propias de las UCI estudiadas, la región y muchos otros factores limitan la inferencia en la toma de decisiones.

\section{CONCLUSIONES}

- La razón de pacientes asignados a una enfermera supera en dos la razón de pacientes asignados a un auxiliar de enfermería

- El evento adverso que con mayor frecuencia se presenta es la neumonía seguida de las infecciones en vías urinarias y el dispositivo médico que con mayor frecuencia se desplaza es la sonda oro gástrica.

- La existencia de una serie de factores contribuyentes llevan a la incidencia de eventos adversos siendo la razón enfermero(a)/ paciente uno de ellos.

- La razón enfermero(a)/paciente establece una relación con la probabilidad de ocurrencia de muerte, paro cardiorrespiratorio, infecciones asociadas con la atención y se puede concluir que para conservar los factores protectores la relación que se debe establecer es de 1 enfermero(a) con un máximo de 4 pacientes críticamente enfermos y un auxiliar con 4 pacientes críticamente enfermos.

- La razón auxiliar de enfermería/paciente guarda relación, desde investigaciones internacionales y el presente estudio, con la incidencia de úlceras por presión y se muestra la presencia de escaras como la variable más sensible dentro de los eventos adversos estudiados para los auxiliares de enfermería.

- Existen otros factores que influyen en la razón enfermero(a) / paciente auxiliar de enfermería / paciente con probabilidad de ocurrencia de los eventos adversos son los ingresos para el riesgo de aumento de mortalidad y los no egresos para el aumento del riesgo de infecciones asociadas con la atención en salud.

- Este estudio confirma los planteamientos establecidos por Avedis Donabedian donde la estructura razón enfermera / paciente auxiliar de enfermería / paciente influye en los resultados de la atención en salud.

\section{REFERENCIAS}

1. Aranaz J, Aibar C. Prevalencia de Efectos Adversos en Hospitales de Latino América. IBEAS. 2009. Madrid: Ministerio de Sanidad; Politica Social e Igualdad.; 2010 Consultado: 12 de junio 2013]. Available from: http://www.msc.es/organizacion/sns/planCalidadSNS/docs/INFORME IBEAS.pdf.

2. Aranaz J, Aibar C, Vitaller J, Ruiz P. Estudio Nacional sobre los Efectos Adversos ligados a la hospitalización ENEAS 2005. Informe Febrero de 2006. Madrid: Ministerio de Sanidad y Consumo. Secretaria General de Sanidad. Dirección General de la Agencia de Calidad del Sistema Nacional de Salud; 2006 Consultado: 12 de junio 2013]. Available from: http://www.msc.es/organizacion/sns/planCalidadSNS/pdf/excelencia/opsc sp2.pdf. 3. Colomer Revuelta C, Agra Varela Y, García Díaz MJ, Fernández-Quero Bonilla P. Incidentes y eventos adversos en medicina intensiva. Seguridad y riesgo en el 
enfermo crítico. SYREC 2007. Informe. Mayo 2009 Madrid: Agencia de Calidad del Sistema Nacional de Salud. Ministerio de Sanidad; Politica Social e Igualdad.; 2009 Consultado: 12 de junio 2013]. Available from: http://www.seguridaddelpaciente.es/contenidos/castellano/SYREC.pdf.

4. Aiken LH, Clarke SP, Cheung RB, Sloane DM, Silber JH. Educational levels of hospital nurses and surgical patient mortality. JAMA. 2003;290(12):1617-23.

5. Aiken LH, Clarke SP, Sloane DM. Hospital staffing, organization, and quality of care: cross-national findings. International Journal for Quality in Health Care. 2002;14(1):5-14.

6. Aiken LH, Clarke SP, Sloane DM, Lake ET, Cheney T. Effects of hospital care environment on patient mortality and nurse outcomes. Journal of Nursing Administration. 2008;38(5):223.

7. Aiken LH, Clarke SP, Sloane DM, Sochalski J, Silber JH. Hospital nurse staffing and patient mortality, nurse burnout, and job dissatisfaction. JAMA. 2002;288(16):1987-93.

8. Aiken LH, Clarke SP, Sloane DM, Sochalski JA, Busse R, Clarke H, et al. Nurses' reports on hospital care in five countries. Health affairs. 2001;20(3):43-53.

9. Aiken LH, Sloane DM, Cimiotti JP, Clarke SP, Flynn L, Seago JA, et al. Implications of the California nurse staffing mandate for other states. Health Services Research. 2010;45(4):904-21.

10. Needleman J, Buerhaus P, Mattke S, Stewart M, Zelevinsky K. Nurse-staffing levels and the quality of care in hospitals. New England Journal of Medicine. 2002;346(22):1715-22.

11. Needleman J, Buerhaus PI. Nurse Staffing and Patient Outcomes in Hospitals Executive Summary. Boston: Harvard School of Public Health; 2001.

12. Beglinger JE. Quantifying patient care intensity: an evidence-based approach to determining staffing requirements. Nursing Administration Quarterly. 2006;30(3):193202.

13. Gerdtz M, Nelson S. 5-20: a model of minimum nurse-to-patient ratios in Victoria, Australia. Journal of Nursing Management. 2007;15(1):64-71.

14. Ramos Domínguez BN. Calidad de la atención de salud: Error médico y seguridad del paciente. Revista Cubana de Salud Pública. 2005;31(3):239-44.

15. Bergmann J. Legislative developments: California enacts nurse-to-patient ratio law. The Journal of law, medicine \& ethics. 1999;27(4):387.

16. Coffman JM, Seago JA, Spetz J. Minimum nurse-to-patient ratios in acute care hospitals in California. Health Affairs. 2002;21(5):53-64.

17. Lang TA, Hodge M, Olson V, Romano PS, Kravitz RL. Nurse-patient ratios: a systematic review on the effects of nurse staffing on patient, nurse employee, and hospital outcomes. Journal of Nursing Administration. 2004;34(7-8):326-37.

18. Borja-Aburto VH. Estudios ecológicos. Salud Pública de México. 2000;42(6):533-8.

19. Manrique-Abril F. Epidemiología social y salud pública. Tunja: FESAD UPTC; 2005. $235 \mathrm{p}$.

20. Navarro JM. Revisión de los estudios sobre los profesionales de enfermería de las Unidades de Cuidados Intensivos de España. Enfermería Global. 2012;11(2):267-89.

21. Mattke S, Needleman J, Buerhaus P, Stewart M, Zelevinsky K. Evaluating the role of patient sample definitions for quality indicators sensitive to nurse staffing patterns. Medical Care. 2004;42(2):21-33.

22. Morrison AL, Beckmann U, Durie M, Carless R, Gillies DM. The effects of nursing staff inexperience (NSI) on the occurrence of adverse patient experiences in ICUs. Australian Critical Care. 2001;14(3):116-21.

23. Numata Y, Schulzer M, Van der Wal R, Globerman J, Semeniuk P, Balka E, et al. Nurse staffing levels and hospital mortality in critical care settings: literature review and meta-analysis. Journal of Advanced Nursing. 2006;55(4):435-48. 
24. Sales A, Sharp N, Li Y-F, Lowy E, Greiner G, Liu C-F, et al. The association between nursing factors and patient mortality in the Veterans Health Administration: The view from the nursing unit level. Medical Care. 2008;46(9):938-45.

25. Rassin M, Silner D. Trends in nursing staff allocation: the nurse to patient ratio and skill mix issues in Israel. International Nursing Review. 2007;54(1):63-9.

26. Garretson S. Nurse to patient ratios in American health care. Nursing Standard. 2004;19(14-16):33-7. 